\title{
Moving from case reports to images in urogynecology
}

\author{
Paul Riss · Peter L. Dwyer • Steven Swift
}

Published online: 12 September 2014

(C) The International Urogynecological Association 2014

Case reports have always been an integral part of the International Urogynecology Journal. Even in the era of evidence-based medicine, case reports have value: readers like them because they widen their clinical horizon and help them sharpen their diagnostic skills [1,2]. Authors, early in their careers, often submit case reports as an entry into publishing manuscripts, and they often find case reports an opportunity to publish their own clinical experience. In addition, most readers enjoy case reports because they are interesting takes on unique issues in clinical practice. Journals, on the other hand, are often ambivalent in regard to case reports. Case reports take up space in the printed issue and are rarely cited, thus affecting the impact factor negatively. As a consequence, journals do not publish case reports at all, limit the number they publish in an issue, or group them together in a special issue or supplement [3]. Some publishers also offer the option to transfer case reports for publication in a separate special open-access journal.

At the International Urogynecology Journal we are aware of these issues and have tried to limit the number of case reports we accept for publication. As a consequence, in 2013, out of the 73 case reports we received only 20 were accepted for publication $(27 \%)$. We have also considered various options. After the successful introduction of the "IUJ Video" section, we think that a way forward could be twofold: we would encourage authors to present a series of cases rather than a single case, with discussion of the relevant literature (published as original articles or reviews). Additionally, we

P. Riss $(\bowtie)$

Department of Obstetrics and Gynecology, Medical University

Vienna, Waehringer Guertel 18-20, 1090 Vienna, Austria

e-mail: priuj11@gmail.com

\section{P. L. Dwyer}

Department of Urogynaecology, Mercy Hospital for Women and Melbourne University, Melbourne, Australia

S. Swift

Department of Obstetrics and Gynecology, Medical University of South Carolina, SC29425 Charleston, USA would create a new section: "Images in Urogynecology", which would take the place of individual case reports.

There is no doubt that with digital technology the use of images has increased dramatically in social and professional media. Many medical journals have special sections for images, with some text, to illustrate a teaching point or a special or unusual case. Case reports most frequently include illustrations, so it seems to us a logical step to invite authors to submit their clinical experience as images with some text and explanation of the pictures. The new section will follow the proven format already in use by other journals and familiar to readers $[4,5]$. An image in urogynecology will consist of one to three pictures (photographs, diagrams, illustrations) with up to 300 words of text and up to three literature references. Each image must be accompanied by a legend providing further information on the case or technique presented. A short video clip could be added as electronic supplemental material (ESM) but videos must be submitted separately for the "IUJ Video" section. All submissions for the "Images in Urogynecology" section will be peer reviewed and managed by one of our editors. Requirements are summarized in the "Instruction for Authors" of the IUJ.

We trust this new section will enhance the value of the IUJ. The final image will always be in color in the digital format. Readers should find the new section attractive and relevant. We invite authors to share with us new techniques or special or unusual cases in this new format.

\section{References}

1. Scott JR (2009) In defense of case reports. Obstet Gynecol 114:413-414

2. Chescheir NC (2013) Case reports deserve respect. Obstet Gynecol 122(2):433-434

3. Hurd WW (2014) Case reports in the era of evidence-based medicine. Obstet Gynecol 124(2):409-410

4. Lopez-Fernandez JA, Marcos-Sanmartin J, Martinez-Escoriza JC (2014) Septate uterus: benign right and malignant left. Am J Obstet Gynecol 211:179, e1-2

5. Zink A, Ring J (2014) Phototoxic dermatitis. N Engl J Med 371:559 